\title{
Propafenone Induced 1:1 Atrial Flutter Conduction
}

Ajay K Sharma*, Sarju Ganatra, Kashif Chaudhry and Muqtada G Chaudhry

Department of Cardiovascular Medicine, Lahey Hospital Medical Center, Burlington, Massachusetts, 01805, USA

${ }^{*}$ Corresponding author: Dr. Ajay K Sharma, Department of Cardiovascular Medicine, Lahey Hospital Medical Center, Burlington, Massachusetts, 01805, USA, Tel: 718-736-5753; E-mail: ajay.sharma@lahey.org

Received date: May 30, 2018; Accepted date: June 20, 2018; Published date: June 28, 2018

Copyright: $\odot 2018$ Sharma AK, et al. This is an open-access article distributed under the terms of the Creative Commons Attribution License, which permits unrestricted use, distribution, and reproduction in any medium, provided the original author and source are credited.

\begin{abstract}
A sixty-seven year old male with a past medical history of diabetes, hypertension, hypercholesterolemia, lung cancer in remission and COPD was diagnosed with symptomatic atrial fibrillation (AF) a month prior to his admission at our institution. He underwent external cardioversion at that time and started on beta blocker, but that had to be discontinued soon thereafter secondary to fatigue. Patient was then started on long acting Verapamil 120 mg daily and Propafenone $225 \mathrm{mg}$ twice a day.
\end{abstract}

Keywords: Atrial fibrillation; Diabetes; Lung cancer; Hypercholesterolemia

\section{Case Presentation}

Patient presented to our facility with acute onset of profound shortness of breath while climbing stairs associated with lightheadedness but without palpitations or chest discomfort. Emergency medical services (EMS) noted the patient to be in wide QRS complex tachycardia with heart rate of 210 beats per minute (Figure 1A) and SBP of $80 \mathrm{~mm} \mathrm{Hg}$. In view of hemodynamic instability, he was electrically cardioverted with $50 \mathrm{~J}$ shock in the field by EMS to AF (Figure 1B), which subsequently spontaneously converted to sinus rhythm with right bundle branch block (RBBB) (Figure 2) during his hospitalization, similar to baseline ECG. His electrolyte panel and hematologic work up was unremarkable. Propafenone was discontinued.

The differential diagnosis for the rhythm strip included ventricular tachycardia and supraventricular tachycardia with aberrancy or underlying bundle branch block. Subsequent analysis revealed a salient feature of the rhythm strips before and after shock in the field and ECG in sinus rhythm. QRS morphology stayed the same throughout and it had become irregularly irregular after emergent electrical shock. Additionally, no AV dissociation could be detected.

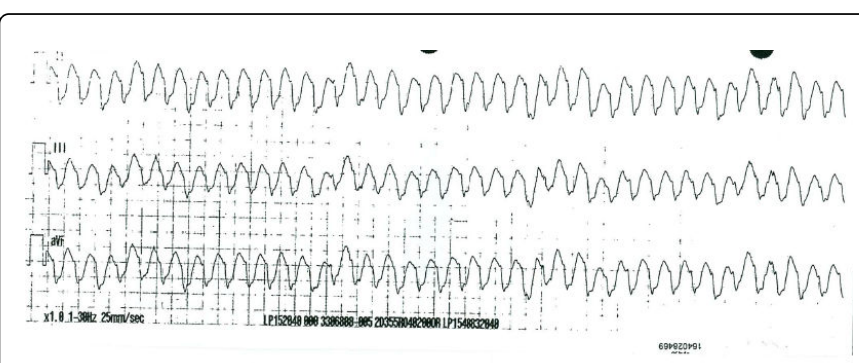

Figure 1A: Initial rhythm strip in field showing wide complex tachycardia.

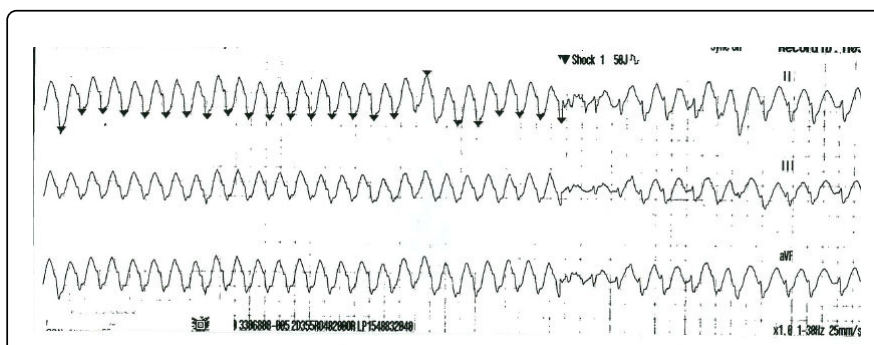

Figure 1B: Rhythm strip before and after shock showing regular wide complex tachycardia degenerating to irregularly irregular wide complex tachycardia.

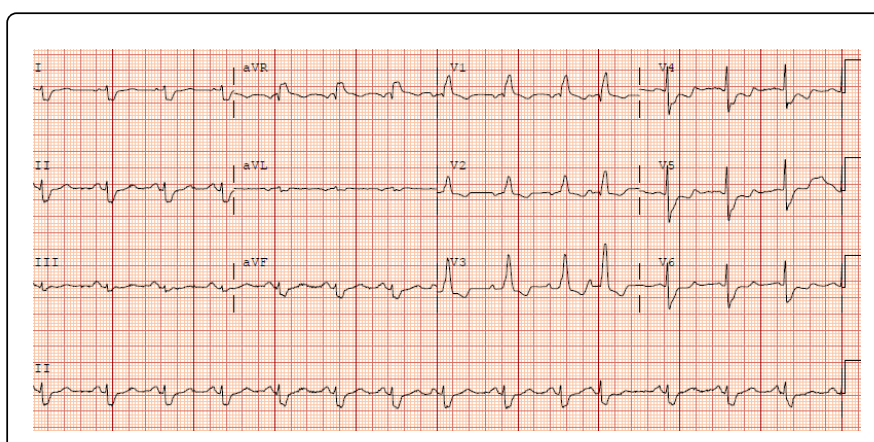

Figure 2: ECG in normal sinus rhythm showing underlying RBBB with similar morphology of QRS complex.

This helped establish diagnosis of atrial flutter with longer than usual cycle length on propafenone with 1:1 conduction to be more likely with degeneration to atrial fibrillation on emergent electrical cardioversion of the initial rhythm was consistent with atrial flutter (AFl) with 1:1 AV conduction and widening of the QRS secondary to the drug effect on infra-nodal conduction. Patient subsequently converted to sinus rhythm spontaneously just prior to the EP study. Successful radiofrequency ablation of cavo-tricuspid isthmus was 
performed. Following ablation, programmed stimulation and rapid atrial pacing failed to induce atrial flutter. There was no evidence of atrial fibrillation during the procedure. The AH interval was $59 \mathrm{msec}$ and HV interval was $67 \mathrm{msec}$.

\section{Discussion}

Propafenone is a class IC antiarrhythmic agent which blocks sodium channel and has weak beta adrenoceptor antagonist activity. ACC/AHA/HRS guidelines recommend reserving its use in patients with symptomatic supraventricular tachycardias (SVTs) (atrioventricular nodal reentrant tachycardia [AVNRT], atrioventricular reentrant tachycardia [AVRT], focal atrial tachycardia [AT]) in patients without structural or ischemic heart disease who are not candidates for, or prefer not to undergo catheter ablation and in whom other therapies have failed or are contraindicated [1].

The mechanism of the 1:1 ventricular response in AFl during administration of antiarrhythmic agent is two-fold:

- A prolongation in the AFl cycle length occurs due to drug-induced decrease in atrial conduction velocity.

- If the atrial rate is slowed sufficiently, the AV node may permit 1:1 AV conduction $[2,3]$.

The ventricular response during AFl is determined by the refractory period of the AV node, the degree of concealed conduction within the node, and the level of autonomic tone. Pre-existing rapid AV nodal conduction can also be a predisposing factor for the occurrence of 1:1 AFl [4]. The association of 1:1 AFl with high catecholamine states, such as exercise, is well documented [5]. AF often converts to macrorentrant AFl with pharmacological therapy; therefore, while administering antiarrhythmic agents for atrial fibrillation, AV nodal conduction-suppressing agents should be used concomitantly.

Class IC agents also have use dependent properties with slow dissociation constant $(>1500 \mathrm{msec})$, whereby at rapid heart rates with abbreviation of diastole, higher concentration of intracellular drug levels can occur, as more of the drug gets into the cell than is uncoupled. As a result, there is markedly decreased velocity in the infra-nodal conduction system that is inscribed as a wide QRS on the surface EKG. Therefore, in patients with 1:1 AV conduction in atrial flutter under the influence of class IC drugs, rapid ventricular rate can mimic ventricular tachycardia with wide and often bizzare looking QRS complexes. This can also lead to considerably dysynchrony in ventricular systolic function with resultant low output state. On the other hand, class III agents show reverse use dependence. These agents usually lengthen the APD at normal and slow heart rates, the prolongation frequently declines as the cycle length is reduced. As a result, the prolongation of APD is marked when it is not needed, but vanishes during tachycardia.
Although 1:1 AV conduction during AFl is thought to be unusual in the absence of antiarrhythmic therapy, there have been a few reports of this phenomenon [6-8]. None of the reported patients had electrocardiographic evidence of pre-excitation, such as an abnormally short PR interval or delta waves during sinus rhythm. However, electrophysiologic studies in such patients suggested that some patients may have had either partial AV nodal bypass tracts with a shorter refractory period than the AV node or an AV node with unusual capacity for rapid conduction $[7,8]$. In our patient, the normal PR and $\mathrm{HV}$ intervals excluded presence of AV bypass tracts.

\section{Conclusion}

Clinicians should be aware of the possibility of "organization" of atrial fibrillation into atrial flutter under the influence of class 1C agents, which is often slower than the usual $300 \mathrm{bpm}$ and can expose those with relatively healthy $\mathrm{AV}$ conduction to the risk of $1: 1 \mathrm{AV}$ conduction with associated wide QRS complexes secondary to usedependent properties of the these drugs on the infra-nodal conduction system and consequently hemodynamic embarrassment.

\section{References}

1. Richard LP, Jose AJ, Mary AC, Hugh C, Jamie BC, et al. (2015) ACC/AHA/HRS Guideline for the Management of Adult Patients With Supraventricular Tachycardia. A Report of the American College of Cardiology/American Heart Association Task Force on Clinical Practice Guidelines and the Heart Rhythm Society. J Am Coll Cardiol 67: p13.

2. Marcus FI (1990) The hazards of using type IC antiarrhythmic drugs for the treatment of paroxysmal atrial fibrillation. Am J Cardiol 66: 366-367.

3. Friedman PL, Stevenson WG (1998) Proarrhythmia. Am J Cardiol 82: 50-58.

4. Kawabata M, Hirao K, Higuchi K, Sasaki T, Furukawa T, et al. (2008) Clinical and electrophysiological characteristics of patients having atrial flutter with 1:1 atrioventricular conduction. Europace 10: 284-288.

5. Kawabata M, Hirao K, Horikawa T, Suzuki K, Motokawa K, et al. (2001) Syncope in patients with atrial flutter during treatment with class Ic antiarrhythmic drugs. J Electrocardiol 34: 65-72.

6. Kennelly BM, Lane GK (1978) Electrophysiological studies in four patients with atrial flutter with 1:1 atrioventricular conduction. Am Heart J 96: 723-730.

7. Aranda JM, Moleiro F, Castellanos A, Befeler B (1975) Electrophysiologic studies in a patient with atrial flutter and 1:1 atrioventricular conduction. Chest 68: 200-204.

8. Castellanos A, Vagueiro MC, Befeler B, Myerburg RJ (1975) Syndrome of short P-R, narrow QRS and repetitive supraventricular tachyarrhythmias: the possible occurrence of the R-on-T phenomenon and the limits of this syndrome. Eur J Cardiol 2: 337-342. 